\title{
EFFECTIVE POPULATION SIZE IN A SONGBIRD: SOME POSSIBLE IMPLICATIONS
}

\author{
MYRON CHARLES BAKER \\ Department of Zoology and Entomology, Colorado State University, Fort Collins, \\ Colorado 80523
}

Received 22.vii. 80

\section{SUMMARY}

\begin{abstract}
Song dialects in White-crowned Sparrows (Zonotrichia leucophrys nuttalli) may act as partial barriers to the free exchange of genes. Within a dialect population, a genetic neighbourhood comprises about 100 individuals. Other factors combine to reduce the effective number to less than 50 . By Wright's methods, the genetic divergence among subpopulations $\left(F_{s t}\right)$ expected in a population exhibiting this size effective number with neutral alleles is about an order of magnitude larger than the observed $F_{s t}$ calculated from electrophoretically detectable alleles. Natural selection may therefore be maintaining greater homogeneity within a dialect than expected by assuming that alleles are neutral. From the torus model of Maruyama, however, the expected $F_{s t}$ values are close to those observed, suggesting no major departures from panmixia within dialect populations. The degree of genetic isolation exhibited between dialect populations may provide an explanation for the more rapid rate of speciation in passerine birds.
\end{abstract}

\section{INTRODUCTION}

NON-PASSERINE birds comprise 84 families and 3518 species or about 42 species per family, whereas passerines are represented by 50 families and 5073 species or about 102 species per family (Mayr and Amadon, 1951). Passerines have about 1.3 subspecies per species and non-passerines about 0.6 subspecies per species (Moreau, 1966). Within the passerines, nonoscines are represented by 14 families and 1083 species or 77 species per family while the oscines (the songbirds, characterized by more than 3 pairs of intrinsic syringeal muscles) comprise 36 families and 3990 species or 111 species per family (Mayr and Amadon, 1951). While there are problems with such crude comparisons these data are consistent with the hypothesis that passerines, in particular oscines, are speciating more rapidly than non-passerines and non-oscines. How can this rapid evolution of songbirds be explained?

The theory that I explore here suggests that part of the explanation for a rapid rate of speciation in songbirds lies in vocal learning: the passerine lineage entered a new adaptive zone characterized by the evolution of vocal learning and vocal learning plays an important role in promoting variability among populations of oscine species by virtue of the occurrence of song dialects. This viewpoint is an extension and variation on separate themes suggested by Fernando Nottebohm (1972) and Guy Bush (Bush et al., 1977).

How might vocal communication play a role in promoting variability? Evolution may be viewed as gene substitution in a population. The probability of fixing a new mutation in a population will depend on several factors acting in concert, but the theory of population genetics indicates that this 
probability is strongly influenced by genetically effective population size (Nei, 1975).

If song dialect populations represent gene pools that are isolated to an important extent, then one could predict divergence in allelic frequencies and observable reduction of dispersal between adjacent dialects. Data consistent with these predictions have been presented previously (Baker, 1975; Baker and Mewaldt, 1978). Yet the dialect population is often extensive in comparison to the distribution of dispersal distances suggesting that a dialect population may not be panmictic. Therefore it is important to examine the genetic substructure of a dialect population by evaluating some influences on genetically effective size.

\section{MethodS AND APPROACH}

The data presented in the theoretical development of this report have been gathered in a population of the non-migratory Nuttall's Whitecrowned Sparrow, Zonotrichia leucophrys nuttalli, occupying a natural environment of scrub vegetation along the central California coast. Life history information on these populations has accumulated from the combined efforts of avian biologists whose activities have centered at the Point Reyes Bird Observatory, near Bolinas, California.

The details of methods employed in studying dispersal, territory size, reproductive success, survivorship, and the ecological genetics of the birds are given in the papers cited in the text. The purpose of the present paper is to weave together the diverse pieces of field information with a theoretical treatment to estimate the genetically effective population size. This estimate is then employed in a discussion of expected and observed genetic heterogeneity in the populations.

\section{Results}

To describe genetic structure I use the concept of isolation by distance in which the basic unit considered is the neighbourhood, defined as the group of individuals whose gametes may come together to form the next generation (Wright, 1946). The size of a neighbourhood in Nuttall's Whitecrowned Sparrow may be derived from a consideration of the distribution of dispersal distances. The dispersal of importance is the distance from the site of fledging of the young bird to that of subsequent breeding and may be called the zygote-to-zygote $(Z Z)$ distance. These dispersal movements in White-crowned Sparrows are strongly linear, corresponding to the geography of the habitat (fig. 1). The suitable breeding area is confined to the coastal scrub zone bounded on one side by the Pacific Ocean and on the other by a forest of Douglas Fir. The strip runs for many kilometers along the coast and averages about one half $\mathrm{km}$ in width in the study area at Point Reyes.

The distribution of $Z Z$ dispersal distances along the axis of the strip of habitat is exponential (Baker and Mewaldt 1978):

$$
Y=\frac{1}{B} e^{-X / B}
$$




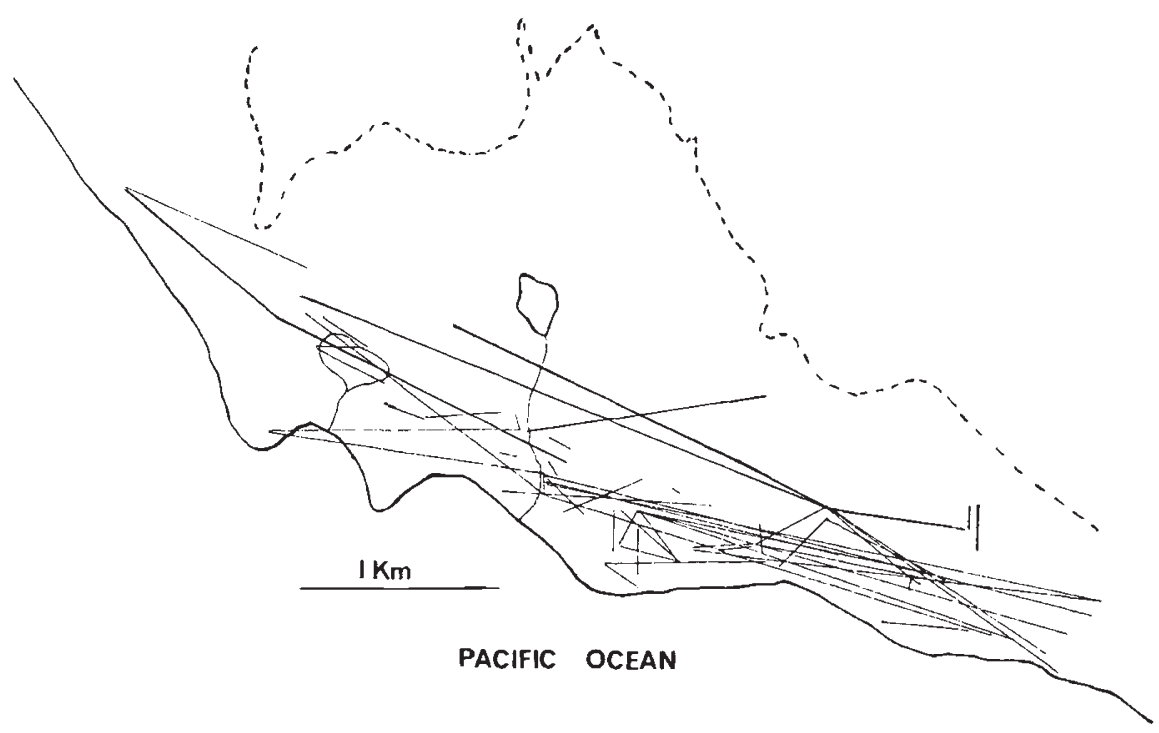

FrG. 1.-Dispersals of individual White-crowned Sparrows banded as fledglings and recaptured as breeding adults. Each line connects the points of first capture and subsequent breeding for individual birds. Short dispersals not included. Dotted line is approximate tree-line.

in which $X$ is the $Z Z$ dispersal distance with mean $B$ estimated by maximum likelihood to be $506 \mathrm{~m}$. Following Wright (1946), to develop the neighbourhood model for this specific application, let $n=$ the number of possible parents in the strip of breeding habitat of length 2B. Wright (1946, p. 40) assumed in his derivation that "the mating system is equivalent to the random union of gametes produced by a population of $N$ monecious individuals, thus involving the proportion $1 / N$ of self fertilization". In an exponential distribution the mean $B$ and standard deviation are equal. Let $D$, density of birds per unit distance, $=n / 2 B$. The average length of a territory is given by the following expression:

$$
\frac{1}{D}=\frac{2 B}{n}=\int_{x_{i}-B / n}^{x_{i}+B / n} \mathrm{~d} x \text {. }
$$

The chance that a gamete is from one individual in the parent generation at distance $X_{i}$ is $Y_{i} / D$. Using $N e$ in place of $N$, the expression to be equated to $1 / N e$ is the probability of two gametes combining from the same individual or:

$$
\sum\left(Y_{i} / D\right)^{2}
$$

Let $N_{e}$ be the effective population size. Thus combining these expressions:

$$
\frac{1}{N_{e}}=\sum_{-\infty}^{+\infty}\left[\frac{2 B}{n} Y_{i}^{2} \int_{x_{i}-B / n}^{x_{i}+B / n} d X\right]=\frac{2 B}{n} \int_{-\infty}^{+\infty} Y^{2} d X .
$$


Substituting into this expression the exponential equation:

$$
\begin{aligned}
\frac{1}{N_{e}} & =\frac{2 B}{n} \int_{-\infty}^{+\infty} \frac{1}{B^{2}} e^{(-2|x|) / B} d X=\frac{2 B}{n B^{2}} \int_{-\infty}^{+\infty} e^{(-2|x|) / B} d X \\
& =\frac{2}{n B}\left\{\int_{-\infty}^{0} e^{(-2|x|) / B} d X+\int_{0}^{+\infty} e^{(-2|x|) / B} d X\right\} \\
& =\frac{2}{n B}\left\{\int_{-\infty}^{0} e^{2 x / B} d X+\int_{0}^{\infty} e^{-2 x / B} d X\right\} \\
& =\frac{2}{n B}\left\{\left.\frac{B}{2} e^{2 x / B}\right|_{-\infty} ^{0}+\left.\left[-\frac{B}{2} e^{-2 x / B}\right]\right|_{0} ^{\infty}\right\} \\
& =\frac{2}{n B}\left\{\frac{B}{2}+\frac{B}{2}\right\}=\frac{2}{n} \\
\frac{1}{N_{e}} & =\frac{2}{n} ; \quad N_{e}=\frac{2 B D}{2}=B D .
\end{aligned}
$$

Density estimates of Z.1. nuttalli in the Point Reyes area indicate considerable variation. One study (Ralph and Pearson, 1971) gave an averaged value for territory size of 0.37 hectares in a year of unusually high density. The authors suggested that this value was probably 50 per cent smaller than normal. In less suitable habitat, territory size is of the order of a hectare. Chamberlain (1972) found territory size to be about 0.76 ha. For present purposes, I take the territory size to be $0.5 \mathrm{ha}$, or approximately $71 \mathrm{~m}$ on a side.

Although the breeding habitat is virtually a strip it does not correspond strictly to a linear model. That is, in a single breeding season, genetic dispersal does not occur across the full width of the strip. On the other hand, the observed dispersal and the narrowness of the strip do not meet the assumptions of area continuity, so it is not correct to employ a bivariate distribution in the development of the equation for $N_{e}$. A reasonable solution toward characterizing the bird density, and one that I have chosen, is to take the average territory width of $71 \mathrm{~m}$ into the strip width of $500 \mathrm{~m}$ giving a value of about 7 territories wide, or 14 breeding adults. Applying this to the linear model gives: $N_{e}=B D=(506 \mathrm{~m})(14 \mathrm{birds}) / 71 \mathrm{~m}=100$ birds. If territory size is $0 \cdot 37$ ha, as suggested by Ralph and Pearson (1971), then $N_{e}=136$ birds. If territory size averages 1 ha, $N_{e}=51$ birds.

Taking the calculated neighbourhood population as 100 birds implies that this is the group of individuals whose gametes may come together. This value of $N_{e}$ is a first approximation of genetically effective size assuming that mating is at random in the neighbourhood population, progeny number is distributed in a poissonian manner, generations are non-overlapping, and population size is constant.

Of these additional influences on $N_{e}$, the last, constant population size, has been considered as adequately as is possible at the present time. Density is known to fluctuate in Z.1. nuttalli as indicated by the territory size measurements mentioned above, but no long-term studies have been done on changes in density. The influence of density change on the value of $N_{e}$ is calculated as a harmonic mean valuc; $N_{e}$ is more strongly infiuenced by low 
densities. The existing data suggest that the estimation of $N_{e}=100$ may be higher than would be found if a long series of estimates were available for calculation.

The effect that variance in progeny number has on $N_{e}$ can be evaluated from existing data (Baker et al., in press). A theoretical study (Crow and Morton, 1955) shows that if progeny number is distributed as a Poisson variate, then the effective number is the same as the actual number of parents. If, however, the variance to mean ratio is greater than unity, then $N_{e}$ is smaller than the actual number of parents.

In Z.1. nuttalli, the Point Reyes data give a value of mean number of nestlings per pair $=5 \cdot 47$, of which $0 \cdot 37$, or 2.02 individuals, will survive to breed, with a variance of 3.42 (Baker et al., in press). As pointed out by Crow and Morton (1955), in a steady state population the mean number of nestlings per pair will equal 2.0 which can be used to adjust the estimates from our data by the following formulation:

$$
\frac{\left(V_{e}-1\right) / 2}{2}=\frac{\left(V_{o}-1\right) / \bar{X}_{o}}{\bar{X}_{o}}
$$

in which $V_{e}=$ expected variance at $\bar{X}=2 \cdot 0, V_{o}=$ observed variance, and $\bar{X}_{o}=$ observed mean. Putting the observed values into the proportion and solving gives the adjusted value of $V_{e}=3.38$ and a variance to mean ratio of 1.69. The equation of Crow and Morton is:

$$
N_{e}=\frac{2 N}{1-F+(1+F)(V / X)}
$$

in which $F$ is the coefficient of inbreeding (Wright, 1949) and is assumed to be zero for the present. Substitution of the variance to mean ratio into this equation gives:

$$
N_{e}=\frac{(2)(100)}{1+1 \cdot 69}=74
$$

The effect of overlapping generations on $\mathrm{Ne}$ is determined by demographic variables (Nei and Imaizumi, 1966). Estimates of the essential parameters for Z.1. nuttalli are available (Baker et al., in press). Nei and Imaizumi derive the equation:

$$
N e=N m \lambda
$$

in which $N m$ is the number born per year that are able to reach the mean reproductive age lambda $(\lambda)$. Lambda is derived from the life table statistics:

$$
\lambda=\frac{\sum 1_{x} m_{x} x}{\sum 1_{x} m_{x}}=\frac{1 \cdot 8}{1 \cdot 0}=1.8 \text { years }
$$

for Z.1. nuttalli. Each pair of parental birds produces about 6.9 eggs in a breeding season, and the probability of an egg reaching the mean age of reproduction is 0.08 approximately. Thus, 37 pairs of White-crowned Sparrows contribute $(37)(6 \cdot 9)(0 \cdot 08)=20$ eggs that are expected to reach lambda. Therefore, $\mathrm{Ne}$ is further reduced from the previous value:

$$
N e=N m \lambda=(20)(1 \cdot 8)=36 \text {. }
$$


At the present time, data on non-random mating in a neighbourhood population are not available. The shape of the dispersal distribution may be used to predict the probability of consanguineous matings of various types (Bulmer, 1973; Greenwood and Harvey, 1978; Cavalli-Sforza et al., 1966) and thus derive a value for $F$, the coefficient of inbreeding. It may be noted that a uniform distribution would suggest random mating but any uneven distribution would suggest a positive $F$ less than or equal to unity.

Z.1. nuttalli, as well as most birds studied, has a dispersal distribution strongly skewed toward the right, suggesting a moderate degree of consanguinity. In view of the highly sedentary tendency of adults, if young stay close, this would increase chances of parent-offspring and brother-sister matings. Other degrees of consanguinity would also occur but contribute much less to $F$. To give some impression of how the size of $F$ would play a role in determining the value of $N_{e}$, in the absence of real data, one can assume a value of $F=0.25$ which is unrealistically high. Using this value in Crow and Morton's equation,

$$
N_{e}=\frac{2 N}{1-F+(1+F)(1 \cdot 69)}=\frac{200}{1-0 \cdot 25+(1 \cdot 25)(1 \cdot 69)}=70,
$$

rather than 74 when $F$ is assumed to be zero. Mating rules based upon social mechanisms could augment or oppose consanguineous matings. Such a mechanism could be based upon song dialects, for example.

To this point, the sexes have been considered together, but it is possible to derived separate values of $N_{e}$ for males and females. $Z$. 1 . nuttalli appears to mate monogamously in the great majority of cases. Some data exist (Petrinovich and Patterson, 1978; Baker, Mewaldt and Stewart, in press) that indicate Z.1. nuttalli has a low level of bigamy, perhaps 10 per cent or less. Using the formulation of Crow and Kimura (1970);

$$
N_{e}=\frac{4 N_{m} N_{f}}{N_{m}+N_{f}}
$$

in which $N_{m}$ is the number of males and $N_{f}$ the number of females, we can see the influence of this level of polygyny. Assume a population of 100 males in which 10 males have 2 females each and the rest are monogamously mated. For this situation $N_{m}=100, N_{f}=120$. Thus:

$$
N_{e}=\frac{(4)(100)(120)}{(100)+(120)}=218
$$

compared to 120 monogamous pairs in which case $N_{e}$ would equal 240. Therefore this level of bigamy suggests a reduction of $N_{e}$ of about 10 per cent.

\section{Discussion}

A potential relationship between vocal learning in songbirds anc' evolutionary processes was pointed out by Marler and Tamura (1962) and developed more explicitly by Nottebohm (1969) with specific reference to the phenomenon of song dialects. The general idea advanced was that song dialects may promote isolation of population subdivisions through mate 
preferences. Concurrently it was demonstrated in laboratory experiments that the dialect elements of song are learned during ontogeny (Marler and Tamura, 1964; Dittus and Lemon, 1969).

There are several speculations regarding the evolution of vocal learning; most workers favour the idea that the explanation involves the greater adaptability of learned characters (Nottebohn, 1972; Immelmann, 1975). A corollary suggests that the larger capacity of the brain for information storage, compared to the genome, allows for more details and therefore more precision in learned preferences. Such adaptability that derives from vocal learning could be expressed in the formation of several kinds of preferences, among which are filial, sexual, species specific, and ecological. Culturally transmitted traits such as learned vocalizations can be studied from an evolutionary perspective in much the same way as genetically inherited traits (Feldman and Cavalli-Sforza, 1976).

The consideration of population size as an influence in evolution has a long history, founded by Sewall Wright $(1943,1946)$ and elaborated and extended more recently (Nei, 1975; Chakraborty and Nei, 1974; Slatkin, 1976). Wilson et al. (1977) used small population size as an explanation for the apparently rapid chromosomal evolution of placental mammals. Quantification of genetically effective population size is lacking for nearly all species of social vertebrates. Although it is tempting to infer small effective size from observations of social organization, this inference is unsubstantiated at the present.

McCraken and Bradbury (1977), for example, found paternity certainty of the male to lie somewhere between the extreme values of 28-94 per cent in harems of the bat Phyllostomus hastatus. Bray et al. (1975) found that in red-winged blackbirds, the harem male may sire only 50 per cent of the offspring produced by his harem females. These values of paternity certainty by harem males represent a major departure from panmixia, but the amount of gene leakage among social units is nevertheless high, perhaps too high to consider the social unit as genetically independent to any important degree.

In the present analysis of White-crowned Sparrows, the final estimation of $N_{e}$ as 36 suggests a considerable potential for evolutionary divergence by random events. A recent analysis of effective population sizes through evolutionary time and a summary of existing data suggests that contemporary vertebrate populations have a deme structure consistent with that during long term evolution, on the order of ten to a few hundred, thus emphasizing the potential effect of random drift on macroevolution (Lande, 1979). Population subdivision in Z.1. nuttalli may occur at the racial and song dialect hierarchial levels as well as at the neighbourhood level. The dialect group is significantly isolated from other dialects, and the effective size of neighbourhoods within a dialect population suggests an additional departure from panmixia. The total effect predicts that Z.1. nuttalli should exhibit strong genetic heterogeneity. The patterns that do exist in electrophoretically detectable enzyme polymorphisms (Baker, 1975) may be evaluated statistically for comparison to the pattern expected for neutral alleles given the calculated value of $N_{e}=36$.

$F$-statistics estimate the genetic heterogeneity in populations. In particular, $F_{s t}$ estimates the amount of genetic differentiation among subpopulations $(s)$ within a total population $(t)$. Calculation of $F_{s t}$ from 
allozyme frequencies within dialects (from Baker, 1975) gives an average $F_{s t}=0 \cdot 0046$. For one comparison with expected values, the theory developed by Maruyama (1972) provides a calculation of genetic divergence that assumes neutral alleles, discrete generations, constant population size, and a normal distribution of dispersal. Using the Maruyama method, and further assuming a mutation rate of $2 \times 10^{-6}, F_{s t}$ for the White-crowned Sparrow was determined to be approximately 0.007 (personal communication from Drs Motoo Kimura and Takeo Maruyama). This amount of genetic divergence is near the observed $F_{s t}$ within dialects found from the allozyme frequencies.

The observed $F_{s t}$ of 0.0046 (from allozyme frequencies) may also be compared to that expected from the effective size of a neighbourhood, calculated earlier to be 36 . The theory for calculating this expected value of $F_{s t}$ is due to Wright (1951). For the neighbourhood analysis derived from the dispersal data, I will take the total population as a dialect group. Dialect populations that I am most familiar with average about $8 \mathrm{~km}$ long by $0.5 \mathrm{~km}$ wide. At a density of 0.5 ha per breeding pair, the dialect population would be 1600 birds. We can take the bracket values of 1000 or 2000 birds as reasonable guesses and compute the expected $F_{s t}$ from the estimate of $N_{e}=36$ by Wright's method. If there are 2000 birds composed of 20 neighbourhoods of 100 birds each, $F_{s t}=0.0569$. If these 20 neighbourhoods are effectively 36 each, $F_{s t}=0 \cdot 0475$. For a dialect population of 1000 birds, we would have 10 neighbourhoods of 100 each giving an $F_{s t}=0.0695$. For 10 neighbourhoods of effective size equal $36, F_{s t}=0.0829$.

Wright's method suggests that there is about an order of magnitude difference $F_{s t}$ values calculated from the neighbourhood analysis and those found directly from enzyme genotypes. The observed genetic structure has less divergence among subpopulations than expected from the neighbourhood analysis. The existing genetic heterogeneity is primarily explained by differences between dialect populations, and $F_{s t}$ values calculated across 4 samples in two dialects have a mean value of $0 \cdot 0123$, more similar to that found from the neighbourhood model.

Analysis by the Maruyama model thus suggests that there is sufficient migration within a dialect to explain the observed genetic heterogeneity. By Wright's neighbourhood model, however, the expected heterogeneity is greater than is observed, arguing that selection may keep allelic frequencies within a dialect more homogeneous than expected from a neutral allele drift model. Thus, in spite of possible subpopulations within dialects, due to viscosity, allelic frequencies within dialect populations may be responding to the average selection regime from the dialect area as a whole. Taken together with the observed allozyme frequency differences between dialect populations, this analysis suggests that song dialect populations may follow relatively independent evolutionary pathways. As a consequence, the genetic variance among populations is elevated, providing the potential for a more rapid rate of speciation in oscine passerines.

Acknowledgements.-I thank Professors James F. Crow, Motoo Kimura, Takeo Maruyama, and Thomas Uzzell for their comments on this paper and Ann E. M. Baker for discussions of ideas contained herein. Professor Maruyama kindly calculated $F_{s t}$ for the torus model. Financial support was provided by National Science Foundation grant DEB-78-22657. 


\section{REFERENCES}

BAKER, M. C. 1975. Song dialects and genetic differences in white-crowned sparrows (Zonotrichia leucophrys). Evolution, 29, 226-241.

BAKER, M. C., AND MEWALDT, L. R. 1978. Song dialects as barriers to dispersal in white-crowned sparrows, Zonotrichia leucophrys nuttalli. Evolution, 32, 712-722.

BAKER, M. C., MEWALDT, L. R., AND STEWART, R. M. Demography of White-crowned sparrows. Ecology, (in press).

BRAY, O. E., KENNELLY, J. J., AND GUARINO, J. L. 1975. Fertility of eggs produced on territories of vasectomized red-winged blackbirds. Wil. Bull., 87, 187-195.

BULMER, M. G. 1973. Inbreeding in the great tit. Heredity, 30, 313-325.

BUSH, G. L., CASE, S. M., WILSON, A. C., AND PATTON, J. L. 1977. Rapid speciation and chromosomal evolution in mammals. Proc. Natl. Acad. Sci. U.S.A., 74, 3942-3946.

CAVALLI-SFORZA, L. L., KIMURA, M., AND BARRAI, I. 1966. The probability of consanguineous marriages. Genetics, 54, 37-60.

CHAKRABORTY, R., AND NEI, M. 1974. Dynamics of gene differentiation between incompletely isolated populations of unequal sizes. Theoret. Pop. Biol., 5, 456-469.

CHAMBERlain, B. 1972. Observations of breeding white-crowned sparrows. Point Reyes Bird Observatory, Newsletter No. 23.

CROW, J. F., AND KIMURA, M. 1970. An Introduction to Population Genetics Theory. Harper \& Row, New York.

CROW, J. F., AND MORTON, N. E. 1955. Measurement of gene frequency drift in small populations. Evolution, 9, 202-214.

DITTUS, W. P. J., AND LEMON, R. E. 1969. Effects of song tutoring and acoustic isolation on the song repertoires of cardinals. Anim. Behav., 17, 523-533.

FELDMAN, M. W., AND CAVALLI-SFORZA, L. L. 1976. Cultural and biological evolutionary processes, selection for a trait under complex transmission. Theor. Pop. Biol, 9, 238-259.

GREENWOOD, P. J. AND HARVEY, P. H. 1978. Inbreeding and dispersal in the great tit. Nature, 271, 52-54.

IMMELMANN, K. 1975. Ecological significance of imprinting and early learning. Ann. Rev. Ecol. Syst., 6, 15-37.

LANDE, R. 1979. Effective deme sizes during long-term evolution estimated from rates of chromosomal rearrangement. Evolution, 31, 234-251.

MARLER, P., AND TAMURA, M. 1962. Song "dialects" in three populations of white-crowned sparrows. Condor, 64, 368-377.

MARLER, P., AND TAMURA, M. 1964. Culturally transmitted patterns of vocal behavior in sparrows. Science, 146, 1483-1486.

MARUYAMA, T. 1972. Rate of decrease of genetic variability in a two-dimensional continuous population of finite size. Genetics, 70, 639-651.

MAYR, E., AND AMADON, D. 1951. A classification of recent birds. Amer. Mus. Novit., No. 1496.

McCRACKEN, G. F., AND BRAdBURy, J. W.. 1977. Paternity and genetic heterogeneity in the polygynous bat, Phyllostomus hastatus. Science, 198, 303-306.

MOREAU, R. E. 1966. On estimates of the past numbers and of the average longevity of avian species. Auk, 83, 403-415.

NEI, M. 1975. Molecular Population Genetics and Evolution. American Elsevier Publ. Co., Inc. New York. 288 pp.

NEI, M., AND IMAIZUMI, Y. 1966. Genetic structure of human populations II. Differentiation of blood group gene frequencies among isolated populations. Heredity, 21, 183-190, 344.

NotTeвонM, F. 1969. The song of the Chingolo, Zonotrichia capensis, in Argentina: Description and evaluation of a system of dialects. Condor, 71, 299-315.

NotтевонM, F. 1972. The origins of vocal learning, Amer. Natur., 106, 116-140.

PETRINOVICH, L., AND PATTERSON, T. L. 1978. Polygyny in the white-crowned sparrow (Zonotrichia leucophrys). Condor, 80, 99-100.

RALPH, C. J., AND PEARSON, C. A. 1971. Correlation of age, size of territory, plumage, and breeding success in white-crowned sparrows. Condor, 73, 77-80.

SLATKIN, M. 1976 The rate of spread of an advantageous allele in a subdivided population. In Population Genetics and Ecology, eds. S. Karlin and E. Nevo. Academic Press, New York.

WILSON, A. C., WHITE, T. J., CARLSON, S. S., AND CHERRY, L. M. 1977. Molecular evolution and cytogenetic evolution. In Human Cytogenetics: ICN-UCLA Symposia on Molecular and Cellular Biology, eds. R. S. Sparkes, D. E. Comings, and C. F. Fox. Academic Press, New York. 
WRIGHT, s. 1943. Isolation by distance. Genetics, 28, 114-138.

WRIGHT, S. 1946. Isolation by distance under diverse systems of mating. Genetics, 31, 39-59. WRIGHT, s. 1949. Adaptation and selection. In Genetics, Paleontology and Evolution, eds.

G. L. Jepson, G. G. Simpson, and E. Mayr. Princeton University Press, Princeton.

WRIGHT, s. 1951. The genetical structure of populations. Ann. Eugenics, 15, 323-354. 